\title{
LIST OF PARTICIPANTS -- IAU COLLOQUIUM 113
}

(Numbers refer to the group photograph; there may be some errors here!)

I. Appenzeller, Heidelberg (16)

L.A. Balona, S.Africa (62)

G.B. Baratta, Rome (58)

B. Bohannan, Colorado (22)

J.P. Cassinelli, Wisconsin (21)

C. Chavarria-K., Mexico City (47)

P.S. Conti, Colorado (33)

M. Corcoran, NASA/Goddard (68)

K. Davidson, Minnesota (63)

M. de Groot, Armagh (49)

C. de Jager, Utrecht (10)

L. Drissen, Montréal (56)

E.L. Fitzpatrick, Princeton

M. Friedjung, Paris (61)

A. Fullerton, Toronto

R. Gabler, Munich (4?)

J.S. Gallagher, Flagstaff (36)

C. Garmany, Colorado

E. Gosset, ESO (35)

H.G. Groth, Munich (2)

B. Hauck, Lausanne (15)

S. Heap, NASA/Goddard (46)

A.G. Hearn, Utrecht (12)

H. Henrichs, Amsterdam

J. Hillier, Harvard-SAO (28)

D.G. Hummer, Colorado (26)

R.M. Humphreys, Minnesota (24)

R.H. Johnson, Oxford (51)

F.D. Kahn, Manchester (3)

J. Klapp, Mexico City (1)

G. Koenigsberger, Mexico City (67)

R.P. Kudritzki, Munich (39)

H.J.G.L.M. Lamers, Utrecht (34)

N. Langer, Göttingen (31)

C. Leitherer, Colorado (7)
M.-C. Lortet, Meudon (40)

A. Maeder, Geneva (32)

J.M. Marlborough, Ontario (6)

S.R. McCandliss, Colorado (42)

P.J. McGregor, Mt. Stromlo (25)

A.F.J. Moffat, Montréal (65)

V. Niemela, Buenos Aires (27)

H. Nieuwenhuijzen, Utrecht (48)

S. Owocki, Delaware (20)

F. Paresce, STSCI, Baltimore

A. Pauldrach, Munich (13?)

R.K. Prinja, London (14)

J. Puls, Munich

C. Robert, Montréal (54)

C. Rossi, Rome

D.D. Sasselov, Toronto

W. Schmutz, Colorado (45)

R.E. Schulte-Ladbeck, Wisconsin (8)

M. Shara, STSCI, Baltimore (23)

S.N. Shore, Socorro (37)

L.J. Smith, London (53)

J. Smolinski, Torun (29)

S.R. Sreenivasan, Calgary (55)

N. St.-Louis, London (17)

O. Stahl, Heidelberg (66)

C. Sterken, Brussels (50)

K. van der Hucht, Utrecht (52)

D. Vanbeveren, Brussels (57)

A. Wagner-Gabler, Munich (5)

N.R. Walborn, STScI, Baltimore (64)

P. Williams, Edinburgh (18)

A.J. Willis, London (41)

B. Wolf, Heidelberg (44)

F.-J. Zickgraf, Heidelberg (19) 